\title{
Radio Detections of Stellar Winds from the Pistol Star and Other Stars in the Galactic Center Quintuplet Cluster
}

\author{
Cornelia C. Lang ${ }^{1,2}$, Don F. Figer ${ }^{2}$, W.M. Goss ${ }^{1}$, and Mark Morris ${ }^{2}$
}

To appear in The Astronomical Journal

\begin{abstract}
VLA images of the Sickle and Pistol H II regions near the Galactic center at 3.6 and $6 \mathrm{~cm}$ reveal six point sources in the region where the dense Quintuplet stellar cluster is located. The spectral indices of five of these sources between $6 \mathrm{~cm}$ and $3.6 \mathrm{~cm}$ have values of $\alpha=+0.5$ to +0.8 , (where $\mathrm{S}_{\nu} \propto \nu^{\alpha}$ ), consistent with the interpretation that the radio sources correspond to ionized stellar winds of the massive stars in this cluster. The radio source associated with the Pistol Star shows $\alpha=-0.4 \pm 0.2$, consistent with a flat or slightly non-thermal spectrum.
\end{abstract}

Subject headings: Galaxy: center-stars: winds-stars: mass loss

\section{Introduction}

High resolution near-infrared (near-IR) observations over the past decade have revealed the presence of massive, unusual stars in the inner $50 \mathrm{pc}$ of the Galaxy, where observations suffer 20-30 visual magnitudes of obscuration. Three clusters of massive stars have been discovered (Glass et al. 1990; Nagata et al. 1990; Okuda et al. 1990; Krabbe et al. 1995; Nagata et al. 1995; Figer et al. 1995, Cotera et al. 1996): (1) the Central cluster, located within a parsec of SgrA*; (2) the Arches cluster, located $\sim 30 \mathrm{pc} \mathrm{N}$ of SgrA* at $\ell=0^{\circ} .12, \mathrm{~b}=0^{\circ} .02$, and (3) the Quintuplet cluster, also located $\sim 35 \mathrm{pc} \mathrm{N}$ of $\operatorname{Sgr}^{*}$, at $\ell=0^{\circ} .16, \mathrm{~b}=0^{\circ} 06$. The stars detected in these clusters have near-IR signatures of OB supergiants and Wolf-Rayet type stars. In the densely populated Quintuplet cluster alone, at least 8 Wolf-Rayet and over a dozen OB supergiants have been discovered. Based on the evolutionary stages of the stars, this cluster is likely to be $3.5 \mathrm{Myr}$ old, with a total estimated mass of $\sim 10^{4} \mathrm{M}_{\odot}$, and a mass density of a few thousand $\mathrm{M}_{\odot} \mathrm{pc}^{-3}$ (Figer et al. 1999a).

The near-IR emission line spectra of stars in the three Galactic center clusters indicate that these stars have evolved away from the zero-age main sequence and have high-velocity stellar winds with terminal wind speeds of 500-1000 $\mathrm{km} \mathrm{s}^{-1}$ (Nagata et al. 1995, Figer et al. 1999a, Cotera et al. 1996, Krabbe et al. 1995, Tamblyn et al. 1996). These powerful winds should be detectable at radio wavelengths, as the radio emission is thermal in nature (i.e., free-free) and arises from the outer parts of the ionized wind envelope. The classic theory of Panagia \& Felli (1975) and Wright \& Barlow (1975) predicts that in the radio regime, the spectrum of wind emission is proportional to $\nu^{+0.6}$ for a spherically symmetric, isothermal, stationary wind expanding at a constant velocity. Previous surveys made with the Very Large Array (VLA) $]^{3}$ have

\footnotetext{
${ }^{1}$ National Radio Astronomy Observatory, Box 0, Socorro, NM 87801, email: clang@nrao.edu

${ }^{2}$ Division of Astronomy, 8371 Math Sciences Building, Box 951562, University of California at Los Angeles, LA, CA 900951562

${ }^{3}$ The VLA is a facility of the National Science Foundation, operated under a cooperative agreement by the Associated Universities, Inc.
} 
detected radio emission arising from the ionized winds surrounding OB supergiants and Wolf-Rayet stars (Abbott et al. 1986; Bieging et al. 1989).

VLA continuum images at $6 \mathrm{~cm}(4.9 \mathrm{GHz})$ and $3.6 \mathrm{~cm}(8.3 \mathrm{GHz})$ of the Sickle and Pistol H II regions near the Galactic center reveal six point sources located in the vicinity of the Quintuplet cluster including the radio source at the position of the Pistol Star (Lang et al. 1997; Yusef-Zadeh \& Morris 1987). The coincidence of the Pistol star in the near-IR with a peak in the $6 \mathrm{~cm}$ radio continuum image was first noted by Figer et al. (1998). In this paper we report that two of the newly identified radio sources in addition to the Pistol Star source are found to be coincident in position with massive stars in the $\lambda=2.05$ $\mu \mathrm{m} H S T$ /NICMOS image of the Quintuplet cluster (Figer et al. 1999b). We discuss the nature of the radio sources and the association with stellar sources in the HST/NICMOS image.

\section{Observations}

\subsection{VLA Continuum Observations}

Table 1 summarizes the VLA continuum images in which the radio point sources are detected. Standard procedures for data reduction and imaging in AIPS have been used in all cases. Both images were made with uniform weighting and have been corrected for primary beam attenuation. The $6 \mathrm{~cm}$ continuum image was made with the data published in Yusef-Zadeh \& Morris (1987), observed with the VLA in the B, C, and $\mathrm{D}$ arrays, and later supplemented with A-array data, to achieve a resolution of $1^{\prime \prime} .33 \times 1{ }^{\prime \prime} 05$, at $\mathrm{PA}=10^{\circ}$.

\section{2. $\quad H S T /$ NICMOS Imaging}

In order to search for stellar counterparts to the radio point sources, a careful alignment was made between the $H S T /$ NICMOS $\lambda=2.05 \mu \mathrm{m}$ image of the Quintuplet cluster (Figer et al. 1999b) and the VLA $8.3 \mathrm{GHz}$ continuum image of this region. The Quintuplet cluster was imaged by HST/NICMOS in a mosaic pattern in the NIC2 aperture (19.' 2 on a side) on UT 1997 September $13 / 14$ in the F205W filter $(\lambda=2.05$ $\mu \mathrm{m})$. The MULTIACCUM read mode with NREADS=11 was used for an effective exposure time of 255 seconds per image. The plate scale was $0{ }^{\prime \prime} 076 \mathrm{pixel}^{-1}$ (x) by $0^{\prime \prime} 075 \mathrm{pixel}^{-1}$ (y), in detector coordinates. The cluster was imaged in a $4 \times 4$ mosaic, and the $+\mathrm{y}$ axis of the detector was oriented $135^{\circ}$ East of North. The images were reduced via the standard NICMOS pipeline (CALNICA, CALNICB; MacKenty et al. 1997) (see Figer et al. 1999b for further details).

A coordinate solution for the HST/NICMOS image was generated by assigning known positions to $\sim 30$ Quintuplet cluster stars using the stellar identifications and coordinates from Figer et al. (1999a), which were obtained using the 3-m Shane telescope at University of California's Lick Observatory. These near-IR positions have an absolute accuracy of $\sim 00^{\prime \prime} .5$. The VLA observations at both $3.6 \mathrm{~cm}$ and $6 \mathrm{~cm}$ have an uncertainty of only $\sim 00^{\prime \prime} 1$, due to signal to noise and the known precision of the calibrator sources. Thus, the alignment of the VLA and HST/NICMOS images has a positional accuracy of $\sigma=0.5$.

\section{Results}




\subsection{Radio Flux Density Measurements}

Figure 1 shows the $3.6 \mathrm{~cm}$ continuum image of Lang et al. (1997) in the vicinity of the Pistol nebula and the Quintuplet cluster. The six radio point sources discussed in this paper are labelled QR1-QR5 and the Pistol Star. The crosses in the image represent the positions of HST/NICMOS sources that are associated with the radio sources; these associations are further discussed below in §3.2.

In previous radio observations, Yusef-Zadeh \& Morris (1987) identified the Pistol nebula as the prominent pistol-shaped source at the center of Figure 1; it has a stellar source near its center of curvature, the Pistol Star (Figer et al. 1998; and references therein). The H92 $\alpha$ recombination line study of Lang et al. (1997) characterizes the Pistol nebula as having an electron temperature of $\mathrm{T}_{e}=3300 \mathrm{~K}$, a complex velocity structure with central velocity near $\mathrm{v}_{L S R} \sim 120 \mathrm{~km} \mathrm{~s}^{-1}$, and extremely broad lines $\left(\Delta \mathrm{v} \sim 60 \mathrm{~km} \mathrm{~s}^{-1}\right)$. In addition, a possible detection of He92 $\alpha$ was made, with a helium to hydrogen abundance, $\mathrm{Y}^{+}=14 \pm 6 \%$. The continuum emission from the Pistol nebula suggests an H II mass of $11 \mathrm{M}_{\odot}$. The absence of molecular material associated with the Pistol nebula, coupled with the low value of $\mathrm{T}_{e}$ compared to other Galactic center H II regions, suggest that this nebula may in fact be the ejecta from a previous stage of the Pistol Star's evolution (Figer et al. 1995; 1998). The source of ionization of the Pistol nebula is primarily due to the radiation field from several of the Quintuplet cluster members (Figer et al. 1995, 1999c; Lang et al. 1997).

With an rms noise level in the $3.6 \mathrm{~cm}$ image of $0.2 \mathrm{mJy}^{\text {beam }}{ }^{-1}$, the six point sources shown in Figure 1 (QR1-QR5 and the Pistol Star source) are detected with S/N ratios between 5 and 10. These sources are also detected at $6 \mathrm{~cm}$ with $\mathrm{S} / \mathrm{N}$ ratios between 5 and 8 . In order to calculate the flux densities at both frequencies, cross cuts were made in both RA and DEC across each point source. Table 2 lists the positions of the point sources, the flux densities at each wavelength, and the spectral index and the deconvolved source size derived from these measurements. The radio sources QR1-QR5 have rising spectral indices, $\alpha=+0.5 \pm 0.4$ to $+0.8 \pm 0.4$, (where $\mathrm{S}_{\nu} \propto \nu^{\alpha}$ ), whereas the Pistol Star has a spectral index of $\alpha=-0.4 \pm 0.2$, consistent with a flat or slightly falling spectrum.

\section{2. $\quad H S T /$ NICMOS Counterparts to VLA sources}

The crosses in Figure 1 show three HST/NICMOS sources (q15, q10, and the Pistol star) which are likely associated with the radio point sources QR4, QR5, and the Pistol Star. The angular offsets in the radio/near-IR positions are $\lesssim 3 \sigma$; the error in the alignment is dominated by the uncertainty in the near-IR positions of $\sigma=0^{\prime \prime} 5$. Figure 2 shows the overlay between the HST/NICMOS image and the $8.3 \mathrm{GHz}$ continuum image (shown in Figure 1). It is also apparent in Figure 2 that three of the radio sources (QR4, QR5 and Pistol Star source) are coincident with HST/NICMOS sources, and that three of the radio sources (QR1, QR2 and QR3) do not have HST/NICMOS counterparts. Given the relatively large surface density of stars in the $H S T$ /NICMOS image of the Quintuplet cluster, the possibility of a chance superposition between a radio source and any HST/NICMOS source is not negligible. However, the probability is much smaller that a randomly placed radio source with a flux density $>1$ mJy $(5 \sigma)$ is coincident with a near-IR source that has been classified as a hot, massive star with a high mass-loss rate (16 sources total in a $77^{\prime \prime} \times$ $74^{\prime \prime}$ region of the HST/NICMOS image; c.f. Figer et al. 1999b). Excluding the Pistol Star as a special case, we calculate that the combined probability that 2 out of 5 radio sources would be randomly aligned (within the $3 \sigma$ positional uncertainty of $1^{\prime \prime} .5$ ) with one of the 16 near-IR supergiants is $4 \times 10^{-5}$. Therefore, it is highly unlikely that these coincidences are due to chance superposition, and indeed represent real 
associations.

\section{Discussion}

\subsection{The Nature of the Radio Sources}

The near-IR counterparts of QR4 and QR5 have been classified as hot, massive stars with high mass-loss rates: q15 has been classified as an OB I supergiant and q10 as WN9/Ofpe, according to Figer et al. (1999a). The radio point sources QR4 and QR5 are presumably detections of the stellar winds arising from the near-IR stars, since their spectra are consistent with $\nu^{+0.6}$ and they have near-IR counterparts. In addition, based on the classic theory of Wright \& Barlow (1975) and Panagia \& Felli (1975), it is possible to predict the radio flux density of the stellar wind arising from an OB supergiant found in the Quintuplet cluster at $3.6 \mathrm{~cm}$. Assuming the following wind parameters (near the extreme values) for an $\mathrm{OB}$ supergiant at the Galactic center - a maximum mass loss rate of $\dot{M}=10^{-4} \mathrm{M}_{\odot} \mathrm{yr}^{-1}$, an electron temperature of $\mathrm{T}_{e}=25,000 \mathrm{~K}$, a terminal wind velocity of $\mathrm{v}_{\infty}=500 \mathrm{~km} \mathrm{~s}^{-1}$, and a distance of $8.0 \mathrm{kpc}$; the predicted radio flux density at $3.6 \mathrm{~cm}$ is $\sim 4 \mathrm{mJy}$. At this frequency, QR1-QR5 have flux densities in the range of 2-6 mJy, consistent with this prediction. Given the rms noise in our images of $0.2 \mathrm{mJy} \mathrm{beam}^{-1}$, we are capable of detecting emission from the winds of OB supergiants in the region of the Quintuplet cluster, and the radio sources are most likely detections of these ionized winds. Since there are at least 8 Wolf-Rayet type stars in the Quintuplet cluster, we can also estimate the radio flux density at $3.6 \mathrm{~cm}$ for these stars, using the following wind parameters: $\dot{\mathrm{M}}=5 \times 10^{-5} \mathrm{M}_{\odot} \mathrm{yr}^{-1}, \mathrm{~T}_{e}=40,000 \mathrm{~K}, \mathrm{v}_{\infty}=2000 \mathrm{~km} \mathrm{~s}^{-1}$, and $\mathrm{d}=8.0 \mathrm{kpc}$; the predicted flux density for a Wolf-Rayet star at the Galactic center is $\sim 0.05 \mathrm{mJy}$ at $3.6 \mathrm{~cm}$. Since the rms noise in both of the VLA continuum maps is 0.2 mJy beam $^{-1}$, we would clearly not have detected the mass-losing Wolf-Rayet stars in the current data, and are only sensitive to the winds arising from OB supergiants.

Although the sources QR1, QR2, and QR3 are detected with $\mathrm{S} / \mathrm{N}>5$, and have spectral indices consistent with stellar wind sources, they have no obvious HST/NICMOS stellar counterparts. A possible explanation is that the near-IR extinction varies across the cluster, and that the stellar counterparts of QR1, QR2 and QR3 are masked by greater extinction than the stellar counterparts of QR4 and QR5. If we invoke extinction to explain the lack of counterparts for QR1-QR3, then a near-IR extinction $\mathrm{A}_{k}>8$ is required, corresponding to a visual extinction $\mathrm{A}_{v}>80$. This kind of extinction is only possible if a dense molecular cloud is located in front of part of the Quintuplet cluster. In that case, the unseen counterparts could still be members of the cluster. However, there is no evidence for such a molecular cloud in this region, which makes this suggestion unlikely.

\subsection{The Pistol Star}

The spectral index of the Pistol Star $(\alpha=-0.4 \pm 0.2)$ is consistent with a flat or slightly falling spectrum. It does not follow the classic theory for a fully ionized wind, which predicts a rising spectrum, $\alpha=+0.6$. The Pistol Star, a prominent source in the near-IR HST/NICMOS image, has been classified as a Luminous Blue Variable (LBV) by Figer et al. (1998) and has a stellar wind. Based on the stellar

parameters for the Pistol Star (c.f., Figer et al. 1998, the "L" model), $\dot{\mathrm{M}}=3.8 \times 10^{-5} \mathrm{M}_{\odot} \mathrm{yr}^{-1}, \mathrm{~T}_{e}=12,000$ $\mathrm{K}, \mathrm{v}_{\infty}=100 \mathrm{~km} \mathrm{~s}^{-1}$, and a distance of $8.0 \mathrm{kpc}$, the predicted radio flux density at $3.6 \mathrm{~cm}$ is $\sim 9 \mathrm{mJy}$ using the formulation of Panagia \& Felli (1973) and Wright \& Barlow (1975). At $3.6 \mathrm{~cm}$, the flux density of the 
Pistol Star is $5.8 \pm 1.0 \mathrm{mJy}$, and at $6 \mathrm{~cm}$ the flux density is $7.4 \pm 1.0 \mathrm{mJy}$.

The radio emission of the Pistol Star source is likely a detection of the ionized wind arising from the Pistol Star. One possible explanation for the slightly falling spectrum is that the Pistol Star may have a non-thermal component in its wind over the cm-wavelength range. This type of spectral index has been observed from other supermassive stars, with $\alpha$ in the range of $\alpha=-0.8$ to 0.0 (Abbott et al. 1984; Persi et al. 1985). In fact, the VLA survey of of Galactic OB stars made by Bieging et al. (1989) finds that $24 \%$ of luminous supergiants are observed to have non-thermal spectra. This fraction is consistent with our results: 1 of the 6 radio point sources we detect has a slightly falling spectral index. Non-thermal emission is thought to arise either by means of shocks in the wind itself, in the shock between the stellar wind and a binary companion (Contreras et al. 1996), or from the interaction of the stellar wind with the remnant of a star's previous evolutionary mass-loss phase (Leitherer et al. 1997).

\section{Conclusions}

Six point sources were detected at $3.6 \mathrm{~cm}$ and $6 \mathrm{~cm}$ with the VLA in the vicinity of the Quintuplet cluster. These sources have rising spectra in the range of $\alpha=+0.5$ to +0.8 , with the exception of the Pistol Star $(\alpha=-0.4)$. Based on the overlay of the HST/NICMOS and $8.3 \mathrm{GHz}$ VLA continuum images, three of these radio sources, including the Pistol Star source, can be identified with hot, massive stars with high-mass loss rates. Therefore, the radio sources are most likely detections of the ionized stellar winds emanating from the supermassive stars in the Quintuplet cluster.

We would like to thank Luis Rodriguez for suggesting that the stellar wind of the Pistol Star may be detectable at $8.3 \mathrm{GHz}$. We would also like to thank Liese van Zee for help with the coordinate solution for the HST/NICMOS image, and Paco Najarro for useful comments on the theory of stellar winds.

\section{REFERENCES}

Abbott, D., Bieging, J.H., \& Churchwell, E., 1984, ApJ, 280, 671

Abbott, D., Torres, A., Biejing, J., \& Churchwell, E. 1986, ApJ, 303, 239

Bieging, J., Abbott, D. \& Churchwell, E. 1989, ApJ, 340, 518

Contreras, M. E., Rodrigues, L., \& Gomez, Y. 1996, ApJ, 469, 329

Cotera, A., Erickson, E., Colgan, S., Simpson, J., Allen, D., \& Burton, M. 1996, ApJ, 461, 750

Figer, D. F., McLean, I., \& Morris, M., 1995, ApJL, 447, L29

Figer, D. F., McLean, I., \& Morris, M. 1999a, ApJ, 514, 202

Figer, D., Kim, S, Morris, M., Serabyn, E., Rich, M., \& McLean, I. 1999b, ApJ, in press

Figer, D. F., Morris, M., Geballe, T. R., Rich, R. M., McLean, I. S., Serabyn, E., Puetter, R., \& Yahil, A. $1999 \mathrm{c}, \mathrm{ApJ}$, in press

Figer, D., Najarro, F., Morris, M., McLean, I., Geballe, T., Ghez, A. \& Langer, N. 1998, ApJ, 506, 384

Glass, I. S., Moneti, A., Moorwood, A. 1990, MNRAS, 242, 69

Krabbe, A., Genzel, R., Eckart, A., Najarro, F., Lutz, D., Cameron, M., Kroker, H., Tacconi-Garman, L.E., Thatte, N., Weitzel, L., Drapatz, S., Geballe, T., Sternberg, A., Kudritzki, R. 1995, ApJL, 447, L95 
Lang, C. C., Goss, W.M., \& Wood, D.O.S. 1997, ApJ, 474, 275

Leitherer, C., Chapman, J., \& Koribalski, B. 1997, ApJ, 481, 898

MacKenty, J. W., et al. 1997, NICMOS Instrument Handbook, Ver. 2.0 (Baltimore: STScI)

Nagata, T., Woodward, C., Shure, M., Pipher, J., and Okada, H. 1990, ApJ, 351, 83

Nagata, T., Woodward, C.E., Shure, M., Kobayashi, N. 1995, AJ, 109, 1676

Okuda, H., Shibai, H., Nakagawa, T., Matsuhara, H., Kobayashi, Y., Kaifu, N., Nagata, T., Gatley, I., \& Geballe, T. 1990, ApJ, 351, 89

Panagia, N. \& Felli, M. 1975, A\&A, 39, 1

Persi, P., Ferrari-Toniolo, M., Tapia, M. Roth, M. \& Rodriguez, L. 1985, A\&A, 142, 263

Tamblyn, P., Rieke, G., Hanson, M., Close, L., McCarthy, D., \& Rieke, M. 1996, ApJ, 456, 206

Wright, A.E. \& Barlow, M.J. 1975, MNRAS, 170, 41

Yusef-Zadeh, F. \& Morris, M. 1987, AJ, 94, 1178 
Table 1. VLA Continuum Image Parameters

\begin{tabular}{ccccc}
\hline \hline$\lambda$ & Resolution & rms noise & Arrays & Reference \\
\hline $3.6 \mathrm{~cm}$ & $2^{\prime \prime \prime} 04 \times 1^{\prime \prime \prime} 71, \mathrm{PA}=59^{\circ}$ & $0.2 \mathrm{mJy} \mathrm{beam}^{-1}$ & DnC, Cn B & Lang, Goss, \& Wood (1997) \\
$6 \mathrm{~cm}$ & $1^{\prime \prime \prime} 33 \times 1^{\prime \prime} 05, \mathrm{PA}=10^{\circ}$ & $0.2 \mathrm{mJy} \mathrm{beam}^{-1}$ & A, B, C, D & Yusef-Zadeh \& Morris (1987) \\
\hline
\end{tabular}

Table 2. Parameters of the Radio Point Sources

\begin{tabular}{|c|c|c|c|c|c|c|c|}
\hline \multirow{2}{*}{$\begin{array}{l}\text { Source } \\
\text { Name }\end{array}$} & \multirow{2}{*}{$\begin{array}{c}\text { R.A. (B1950) } \\
(\text { h m s })\end{array}$} & \multirow{2}{*}{$\begin{array}{c}\text { Decl. }(\mathrm{B} 1950) \\
\left({ }^{\circ},{ }^{\prime \prime}\right)\end{array}$} & \multicolumn{2}{|c|}{ Flux Density (mJy) } & \multirow{2}{*}{$\begin{array}{l}\text { Spectral } \\
\text { Index* }\end{array}$} & \multirow{2}{*}{$\begin{array}{c}\text { Deconvolved } \\
\text { Size }\end{array}$} & \multirow{2}{*}{$\begin{array}{c}\text { NICMOS } \\
\text { Counterpart }\end{array}$} \\
\hline & & & $\lambda=3.6 \mathrm{~cm}$ & $\lambda=6 \mathrm{~cm}$ & & & \\
\hline Pistol Star & $\begin{array}{lll}17 & 43 & 04.77 \pm 0.01\end{array}$ & $-2848 \quad 57.0 \pm 0.1$ & $5.8 \pm 1.0$ & $7.4 \pm 1.0$ & $-0.4 \pm 0.2$ & $3 . \prime 5$ & yes \\
\hline QR1 & $174304.72 \pm 0.01$ & $\begin{array}{lll}-28 & 48 & 14.9 \pm 0.1\end{array}$ & $3.0 \pm 1.0$ & $1.9 \pm 0.4$ & $+0.8 \pm 0.3$ & $2 . \prime 0$ & no \\
\hline QR2 & $\begin{array}{lll}17 & 43 & 04.52 \pm 0.01\end{array}$ & $-28 \quad 48 \quad 13.2 \pm 0.1$ & $5.6 \pm 1.0$ & $3.6 \pm 0.9$ & $+0.8 \pm 0.2$ & $2 . \prime 5$ & no \\
\hline QR3 & $174303.95 \pm 0.01$ & $-28 \quad 48 \quad 12.1 \pm 0.1$ & $3.0 \pm 1.0$ & $2.2 \pm 0.7$ & $+0.8 \pm 0.4$ & $2 .{ }^{\prime \prime} 0$ & no \\
\hline QR4 & $174304.61 \pm 0.01$ & $\begin{array}{lll}-28 & 48 & 22.2 \pm 0.1\end{array}$ & $2.9 \pm 0.9$ & $1.8 \pm 0.6$ & $+0.5 \pm 0.4$ & $3 . \prime 0$ & yes \\
\hline QR5 & $174304.60 \pm 0.01$ & $-2848 \quad 30.0 \pm 0.1$ & $1.3 \pm 0.7$ & $0.8 \pm 0.5$ & $+0.8 \pm 0.6$ & $4 .^{\prime \prime} 0$ & yes \\
\hline
\end{tabular}

${ }^{*} \mathrm{~S} \propto \nu^{\alpha}$ 


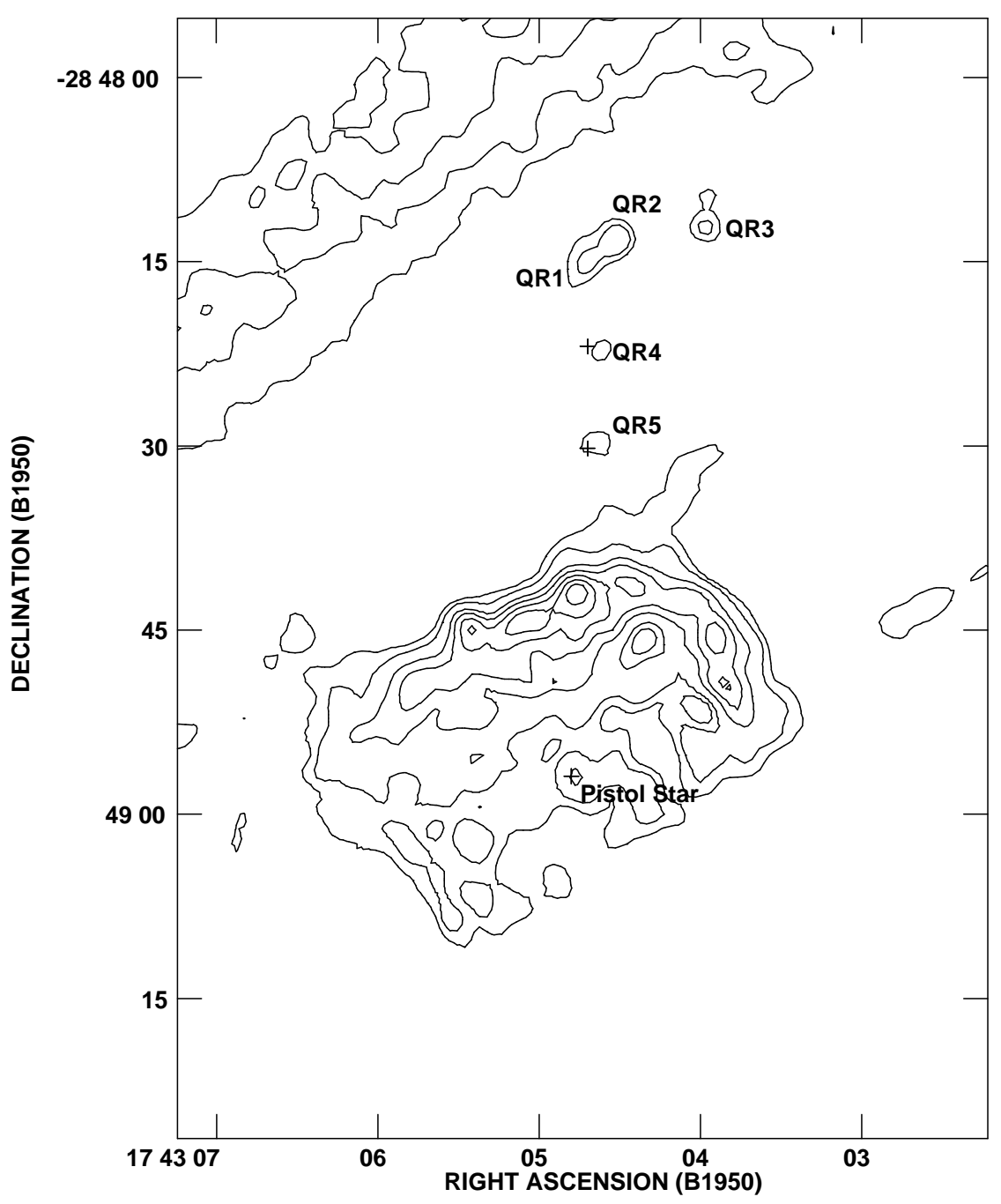

Figure 1 - VLA $3.6 \mathrm{~cm}$ radio continuum image of the six radio point sources detected at both 3.6 and 6 $\mathrm{cm}$, labelled QR1-QR5, and the Pistol Star. This image has a resolution of $2^{\prime \prime} \cdot 12 \times 1^{\prime \prime} .71, \mathrm{PA}=59^{\circ}$. The contours represent $0.5,1,2.5,4,6,8,10,12,14,16,18,20,22 \mathrm{mJy}$ beam $^{-1}$; where $0.5 \mathrm{mJy}_{\text {beam }}{ }^{-1}$ corresponds to $1.5 \sigma$. The crosses represent $H S T$ /NICMOS positions of stars that are associated with three of the radio sources, with positional uncertainties of $\lesssim 3 \sigma$, where $\sigma=0^{\prime \prime} .5$. 


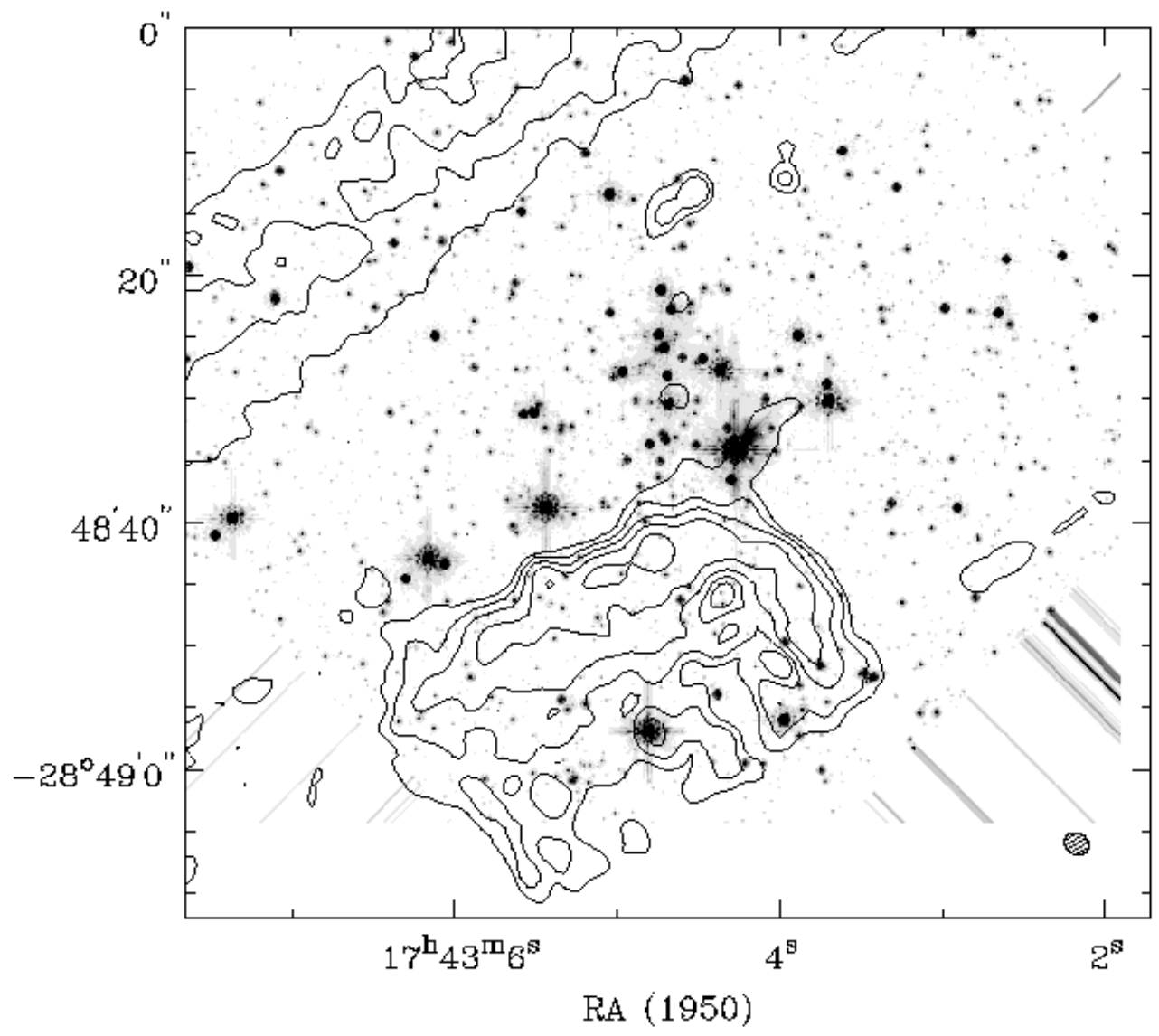

Figure 2 - VLA $3.6 \mathrm{~cm}$ continuum image (as shown in Figure 1) overlaid on $H S T /$ NICMOS $\lambda=2.05 \mu \mathrm{m}$ image of the Quintuplet cluster. 